\title{
PERFIL EMOCIONAL Y COMPETENCIAS MATEMÁTICAS DE LOS ESTUDIANTES DEL GRADO DE EDUCACIÓN PRIMARIA
}

\author{
Rafael Escolano Vizcarra, José María Gairín Sallén \\ Universidad de Zaragoza \\ Clara Jiménez-Gestal, Jesús Murillo Ramón, \\ Luz Roncal Gómez \\ Universidad de La Rioja
}

\begin{abstract}
RESUMEN: El objetivo global de la investigación es continuar los trabajos realizados a lo largo de más de diez años (Hidalgo, Maroto y Palacios, 1999 e Hidalgo, Maroto, Ortega y Palacios, 2008) enfocados al análisis de los factores implicados en el rendimiento de la asignatura de matemáticas. Intentamos ahora completar el estudio de los diferentes niveles educativos, que comenzamos en la Educación Infantil y Primaria, analizando el nivel universitario del que saldrán los futuros maestros de matemáticas. En este artículo, se presentan unos primeros resultados de la investigación para valorar la relación entre el perfil emocional del futuro graduado en Educación Primaria y sus creencias sobre el desempeño de su trabajo como profesor de matemáticas. En la primera parte del artículo se indaga sobre la competencia matemática de estudiantes de primer curso de cuatro universidades españolas, utilizando como instrumento de la competencia matemática dos cuestionarios elaborados con las pruebas liberadas del Informe PISA 2003. En la segunda parte del artículo se estudian las relaciones entre la competencia matemática de los futuros maestros, las creencias de estos estudiantes sobre la naturaleza de las matemáticas y sus creencias sobre su competencia como profesores de matemáticas, utilizando unas escalas elaboradas y validadas en proyectos $\mathrm{I}+\mathrm{D}$ anteriores.
\end{abstract}

PALABRAS CLAVE: Matemáticas, competencia, PISA, enseñanza emocional, maestro.

\section{EMOTIONAL PROFILE AND THE MATHEMATICAL COMPETENCES OF THE FUTURE PRIMARY SCHOOL TEACHERS}

ABSTRACT: The global aim of this research is to continue the work, carried out for more than ten years (Hidalgo, Maroto y Palacios, 1999 e Hidalgo, 
Maroto, Ortega y Palacios, 2008), focused on the analysis of the factors involved in the student's performance concerning the mathematics subject. We try now to complete the study, in the different educational levels, that we started with the Childhood and Primary Education, and we analyze the universitary level and the mathematics teachers-to-be. In this paper, we show the first results that allow us to consider the relationship between the emotional profile of the future Primary School teachers and their beliefs about their work as mathematics teachers. In the first part of the paper, we investigate the mathematical competence of firstyear students in four Spanish universities, using two questionnaires made up with the released tests of PISA Report 2003. In the second part of the paper, we study the relations between the mathematical competence of the future teachers, their beliefs about the nature of mathematics and their beliefs about their competence as mathematics teachers, by using some scales developed and validated in previous I+D projects.

KEYWORDS: Mathematics, competence, PISA, emotional teaching, teacher.

Recibido: $15 / 07 / 2011$

Aceptado: 15/02/2012

\section{INTRODUCCIÓN}

Las leyes orgánicas de educación vigentes en España, tanto en Educación Primaria como en Educación Secundaria Obligatoria, se han hecho eco de la tendencia europea sobre educación matemática que toma como modelo la educación en competencias. Esta misma tendencia se encuentra en los planes de estudio que se acaban de implantar en el Grado de Educación Primaria. A partir de hacerse públicos los resultados de la evaluación PISA 2003, existe abundante literatura sobre educación en competencias.

\subsection{Situación e interés}

Desde que en 1988 el NCTM redactara el primer borrador de los estándares curriculares (declaración de principios sobre qué tiene valor y qué no lo tiene) la percepción sobre la educación matemática que alcanzan los alumnos dio un giro considerable. Los estándares están orientados a la adquisición de potencia matemática (la capacidad que tienen el individuo de explorar, formular hipótesis y razonar lógicamente, así como la capacidad de usar de forma efectiva diversos métodos matemáticos para resolver problemas imprevistos y un desarrollo de la confianza en sí mismo) (N.C.T.M., 1991). Los estándares novedosos son los precursores de una nueva percepción de la educación matemática en Europa que, en la actualidad, toma como modelo la educación en competencias (Rico, L., 2003; Murillo, J. y Marcos, G., 2009). Según INCE (2000, 13), los marcos conceptuales en los que se basan las evaluaciones del proyecto PISA fueron creados por grupos de expertos, entre los que destacan J. de Lange (director), M. Niss y T. Romberg y, como se indica en INECSE (2004), fueron adoptados por los gobiernos de los países de la OCDE en 1998. La primera evaluación tuvo lugar en el año 2000, pero en España, en cierto modo, pasó 
inadvertida, aunque no ocurrió así en otros países, como por ejemplo en Dinamarca, donde Niss (2002) hizo público The Danish KOM Project. La influencia llegó a España con la publicación de los resultados de PISA 2003, destacando las publicaciones del Instituto Nacional de Evaluación y Calidad del Sistema Educativo, INECSE (2004, 2005), que hacen públicos los problemas liberados y los marcos teóricos y el estudio internacional de tendencias en Matemáticas y Ciencias, y con el informe TIMSS 2003, recogido en Mullis et al. (2002). Las últimas leyes orgánicas de educación se han hecho eco de esta nueva orientación y, sin olvidar la educación en la atención a la diversidad, se han formulado en términos de competencias, constituyendo los dos pilares curriculares de la educación matemática, tanto en Educación Primaria como en Educación Secundaria Obligatoria. En los últimos años han aparecido numerosos trabajos de reflexión e investigación en nuestro país, unos han sido publicados por el Ministerio de Educación, otros por las Comunidades Autónomas (sobre todo, por las que han obtenido mejores resultados) y, otros por prestigiosos eruditos en Didáctica de la Matemática. Las publicaciones de las dos primeras son de dominio público, sobre todo las del Ministerio de Educación, ya que el Instituto de Evaluación tiene publicados estos estudios en su página web. En menor escala aparecen las publicaciones de las Consejerías de Educación (Castilla León, Cataluña, Navarra, País Vasco, La Rioja) que ciertamente son menos interesantes ya que, de alguna manera, en todas ellas aparece cierto sesgo de los datos. De los trabajos realizados por expertos de Didáctica de la Matemática relacionados con PISA, sobre todo con PISA 2003, (muchos de ellos aparecen publicados en la red y son de acceso libre), entre otros muchos citamos a: Rico y Lupiañez (2008), quienes basándose en un enfoque funcional de las matemáticas curriculares, facilitan una aproximación racional a las competencias matemáticas desde una perspectiva curricular; Calleja, Ortega, Calleja, Arias y Crespo (2007) determinan que la educación matemática en Castilla y León no se produce en términos de competencias; Rico (2005a) analiza el término de competencia y las principales componentes del marco PISA, Rico (2005b) indica que parte del fracaso de los alumnos debe imputarse a deficiencias estructurales y de formación de profesorado; Rico (2008), González y Lupiañez (2005) analizan el valor social del conocimiento matemático desde la perspectiva de PISA 2003; De León y Recio (2005) estudian la presencia de las matemáticas en la vida real; Flores y Moreno (2005) exploran la participación de la familia y de los agentes sociales en la educación matemática; Solar, Azcárate y Deulofeu (2008) proponen una caracterización de competencias sobre la práctica educativa de las gráficas de las funciones; Moreno, Mesa, y Azcárate (2007) construyen una plantilla para evaluar los textos de Análisis Matemático desde la perspectiva de las competencias y los niveles de evaluación de PISA 2003.

Fuera de España, en los últimos años, no se han prodigado tantos estudios sobre competencia matemática, de hecho en la base de datos Mathdy de ZDM apenas se encuentran referencias a investigaciones sobre el tema, pero, sin duda, el que más ha influido en España ha sido OCDE (2004), que presenta las concepciones y el diseño del ciclo de evaluación PISA 2003 centrado en las Matemáticas, conteniendo el detalle de la fundamentación teórica y el diseño de este ciclo de evaluación junto con ejemplos de las preguntas propuestas (INECSE (2004a) es la traducción que ha publicado el MEC). 
La formación inicial del Profesorado de Matemáticas ha sido y es un tema de investigación muy importante en España. Concretamente, la Sociedad Española de Investigación en Educación Matemática SEIEM creó y acoge al Grupo Nacional de Investigación en Formación de Profesorado y, como describe Llinares (2008), sus investigaciones se han prodigado en los últimos años en dos agendas diferentes: por una parte, el interés se ha centrado en el aprendizaje de los contenidos matemáticos y de destrezas útiles para enseñar y, por otra, las relaciones entre teoría y práctica como elemento de reflexión y análisis. Entre las más relevantes de los últimos años, destacamos las siguientes: Estrada, Batanero y Fortuny (2005) analizan el conocimiento que sobre estadística elemental tienen los profesores en formación; Peñas y Flores (2005) investigan procesos de reflexión de estudiantes para profesores de matemáticas; Araujo, Giménez y Rosich (2006) realizan un estudio sobe afectos y demostraciones geométricas en estudiantes para maestros; Barrantes y Blanco (2006) hacen un estudio sobre concepciones en geometría; García, Sánchez, Escudero y Llinares (2006) analizan la relación entre investigación y práctica en profesores de matemáticas; Carrillo, Climent, Contreras y Muñoz-Catalán (2007) analizan el desarrollo profesional de los profesores de matemáticas a través de un modelo cognitivo; Llinares y Vals (2007) analizan cómo se adquiere el conocimiento de la enseñanza de las matemáticas en los profesores de Educación Infantil.

En otros países, Wedege (2004) utiliza una metodología en la investigación basada en un modelo de competencias para analizar la enseñanza de las matemáticas y la educación de adultos sobre aritmética elemental, metodología que resultó ser útil en la formación docente; Lawson (2004) utiliza los datos de pruebas de diagnóstico en la Universidad de Conventry para ilustrar cómo la entrada de estudiantes en las competencias básicas matemáticas ha cambiado durante el período 1991-2001 y, según el autor, ha habido una disminución significativa en muchos conocimientos matemáticos, que son considerados por la educación superior como elemento esencial para aquellos que realizan cursos de grado con un contenido matemático. Winslow (2005) analiza las competencias de los profesores de matemáticas (Currículo, enseñanza, descubrimiento y aprendizaje, evaluación, colaboración, desarrollo profesional) y su desarrollo durante el período de prácticas, y plantea importantes desafíos al sistema educativo danés. Perels, Guertler y Schmitz (2005) concluyen que es posible mejorar la resolución de problemas matemáticos y la autorregulación a través de la correspondiente competencia y muestran que la combinación de autorregulación y estrategias de resolución de problemas lleva a la mejora de las competencias de autorregulación; Spinath, B., Spinath, F. (2005) investigan la relación entre la enseñanza general y el aprendizaje, contrastando motivación, creencias y competencia, encontrando que la competencia de los niños estaba moderadamente asociada a creencias y fuertemente asociada con la motivación de su aprendizaje. Singer, en un primer artículo (2006), describe un modelo cognitivo para la adquisición de un currículo basado en competencias $y$, posteriormente (2009), considera que un factor clave en el aprendizaje del lenguaje y de las matemáticas es desarrollar un currículo basado en competencias que destaquen los diversos tipos de trasferencias. Finalmente, Shoenfeld (2008ab) y Milgram (2008) tratan aspectos interesantes sobre la evaluación para nuestra investigación. 


\subsection{La noción de competencia matemática}

En el marco del proyecto PISA, la competencia matemática es la aptitud de un individuo para identificar y comprender el papel que desempeñan las matemáticas en el mundo, alcanzar razonamientos bien fundados y utilizar y participar en las matemáticas en función de las necesidades de su vida como ciudadano constructivo, comprometido y reflexivo. (INECSE 2004b, p. 5). PISA examina las competencias en lectura, matemáticas y ciencias. En cada ciclo de PISA se examinan siempre estas tres competencias pero una de ellas con especial relevancia. El formato de las preguntas puede ser cerrado o abierto. Las preguntas cerradas adoptan la forma de respuesta múltiple en la que el alumno ha de escoger una sola de las opciones de respuesta presentadas (normalmente cuatro). Las preguntas abiertas se contestan redactando o calculando la respuesta en un espacio abierto. Las puntuaciones sufren un proceso de escalamiento según la metodología TRI (Teoría de Respuesta al Ítem) y la calificación global se expresa en unidades de una escala con media de 500 puntos y desviación típica de 100. Tanto los alumnos como las preguntas reciben una puntuación en la escala PISA. En el caso de los alumnos las puntuaciones más altas significan una mayor competencia, mientras que en el caso de las preguntas una mayor dificultad, entendida como menor probabilidad de obtener una respuesta correcta.

PISA, en Matemáticas mide el rendimiento de los alumnos en cuatro subáreas matemáticas:

- Espacio y forma, que engloba los fenómenos espaciales y geométricos y las propiedades de los objetos.

- Cambio y relaciones, que engloba las relaciones entre variables y la comprensión de los modos en que se representan, lo que incluye las ecuaciones.

- Cantidad, que engloba los fenómenos numéricos, así como los patrones y las relaciones cuantitativas.

- Incertidumbre, que engloba los fenómenos estadísticos y de probabilidad.

Los resultados que presentamos, corresponden a las pruebas de ítems de PISA tanto de Matemáticas como de Resolución de Problemas y a las creencias sobre la naturaleza de las matemáticas y su competencia profesional de 334 alumnos de los estudios de Magisterio de diversas Comunidades Autónomas. Las puntuaciones posibles, en nuestro estudio, oscilan entre 0 y 2 puntos por ítem, siempre en unidades enteras, sin decimales. Una respuesta errónea obtiene 0 puntos. Las preguntas de respuesta cerrada, tienen una puntuación máxima de 1 punto y las preguntas abiertas reciben una puntuación máxima de 2 puntos o una puntuación parcial de 1 punto. Los criterios de corrección fundamentalmente son los utilizados en PISA.

Como objetivos, nos planteamos los siguientes:

1. Pretendemos, mediante un cuestionario elaborado con las pruebas liberadas en el Informe PISA (2003), conocer la competencia matemática de los alumnos que comienzan los estudios de grado de Maestro de Educación Primaria.

2. Estudiar las relaciones entre la competencia matemática y las creencias de los estudiantes sobre la naturaleza de las matemáticas y sobre su competencia como profesores de matemáticas en Educación Primaria. 


\section{Resultados en las cuatro subáreas de matemáticas}

Se ha recogido información que permite distinguir las cuatro subáreas dentro de la exploración de la competencia matemática. PISA para determinar el nivel de competencia matemático, establece los niveles de complejidad de acuerdo con los resultados de la evaluación realizada. De este modo se determinan empíricamente seis niveles de competencia, que admiten una descripción general y también una descripción por cada uno de los campos de contenido. Se señalan la media y el error típico para cada una de estas subescalas en la Tabla 1. Los alumnos españoles se muestran relativamente menos competentes en las subáreas de Espacio y forma (476 puntos) y Cambio y relaciones (481). Se muestran más fuertes en la de Incertidumbre (489) y, sobre todo, en la de Cantidad (492). El conjunto de alumnos de los países de la OCDE se muestra en los mismos términos en las mismas subáreas, aunque la máxima puntuación la obtienen en Incertidumbre mientras que los españoles lo hacen en Cantidad.

En Resolución de problemas: Promedio OCDE 500 (0,6). Promedio España 482 $(2,7)$.

\section{Tabla 1}

\begin{tabular}{|l|c|c|c|c|}
\hline SUBESCALAS & $\begin{array}{c}\text { Espacio y forma } \\
\text { (Geometría) }\end{array}$ & $\begin{array}{c}\text { Cambio } y \\
\text { relaciones } \\
\text { (Álgebra) }\end{array}$ & $\begin{array}{c}\text { Cantidad } \\
\text { (Aritmética) }\end{array}$ & $\begin{array}{c}\text { Incertidumbre } \\
\text { (Estadística y } \\
\text { Probabilidad) }\end{array}$ \\
\hline OCDE & $496(0,6)$ & $499(0,7)$ & $501(0,6)$ & $502(0,6)$ \\
\hline$E S P A \tilde{N} A$ & $476(2,6)$ & $481(2,8)$ & $492(2,5)$ & $489(2,4)$ \\
\hline
\end{tabular}

Se han pasado a nuestros alumnos dos tipos de cuestionarios, el A y el B, cada uno aproximadamente al 50\%. Se presentan a continuación la ordenación de los distintos ítems pasados a nuestros alumnos en las dos pruebas, según niveles de dificultad basados en los porcentajes de éxito.

\subsection{Ordenación resultados, según porcentajes de éxito}

Se proporciona el porcentaje de éxito de nuestros alumnos en cada uno de los ítems, junto con los obtenidos en el conjunto de la OCDE y de España en la Tabla 2. Presentamos gráficamente los resultados obtenidos, indicado cual es la estrategia seguida por los alumnos en la resolución del ítem correspondiente en la Figura 1. Los dos ítems con menor nivel de dificultad, Crecer-2 y Coche-1, presentan un porcentaje de éxito superior al 90\%. En el primer caso es interesante destacar que cerca del $20 \%$ de los alumnos que plantean una solución correcta lo hacen, bien de manera incompleta, bien utilizando un lenguaje "no matemático" (Figura 2). Los ítems Niveles y Chatear fueron respondidos con éxito por más del $80 \%$ de los alumnos, si bien en torno al 13\% de las respuestas correctas en Niveles se consideró incompleta (Figura 3). En ambos casos el porcentaje de éxito se aproxima 
al $70 \%$ y cerca de la cuarta parte de las respuestas evaluadas son incorrectas (Figura 4). Tanto en los aciertos como en los fracasos de los ítems Robos y Crecer-1, aparecen diferentes estrategias. Se han destacado las de resolución correcta. En el caso de Robos, se observa que mayoritariamente las respuestas son incompletas, mientras que en Crecer- 1 lo más destacable es la respuesta correcta en lenguaje cotidiano (Figura 5). En estos dos casos destaca el nivel de fracaso, en torno al $60 \%$. Esto en el caso de Carpintero muestra la persistencia de la falta de competencia en Geometría. En el caso de Vuelo, las respuestas obtenidas nos sugieren la clasificación del mismo como de Nivel 6, de dificultad máxima (Figura 6). Lo más destacable que refleja esta figura es el alto grado de fracaso, que muestra la falta de competencia en la resolución de problemas que se mantiene en nuestros alumnos estudiantes de Maestro.

\section{Tabla 2}

\begin{tabular}{|c|c|c|c|c|c|c|c|}
\hline \multicolumn{5}{|c|}{ Alumnos de la prueba } & \multirow{2}{*}{$\begin{array}{c}\text { Nuestros } \\
9,5\end{array}$} & \multirow{2}{*}{$\frac{O C D E}{28,10 \%}$} & \multirow{2}{*}{$\begin{array}{l}\text { ESPAÑA } \\
19,20 \%\end{array}$} \\
\hline Cambio & Crecer-2 & n de 1 & 16 & $\%$ & & & \\
\hline & & $n \mathrm{de} 2$ & 150 & $\%$ & 89,3 & $54,70 \%$ & $62,40 \%$ \\
\hline & & n de 0 & 2 & $\%$ & 1,2 & & \\
\hline \multirow{2}{*}{ cambio } & Coche-1 & n de 1 & 153 & $\%$ de aciertos & 92,2 & $72,90 \%$ & $71,40 \%$ \\
\hline & & n de 0 & 12 & $\%$ de fallos & 7,2 & & \\
\hline \multirow[t]{3}{*}{ cantidad } & Niveles & nde 1 & 22 & $\%$ & 13,1 & $5 / \mathrm{V}$ & $s / v$ \\
\hline & & nde 2 & 117 & $\%$ & 69,6 & & \\
\hline & & nde 0 & 29 & $\%$ & 17,3 & & \\
\hline \multirow{2}{*}{ cambio } & Chatear & n de 1 & 135 & $\%$ de aciertos & 81,3 & $53,70 \%$ & $46 \%$ \\
\hline & & nde 0 & 31 & $\%$ de fallos & 18,7 & & \\
\hline & & & & & & & \\
\hline \multirow[t]{2}{*}{ incertidumbre } & Puntuaciones & nde 1 & 118 & $\%$ de aciertos & 70,2 & $32,20 \%$ & $27,80 \%$ \\
\hline & & nde 0 & 50 & $\%$ de fallos & 29,8 & & \\
\hline & & & & & & & \\
\hline \multirow[t]{2}{*}{ cambio } & Coche-2 & $n$ de 1 & 114 & \% de aciertos & 68,7 & $25,40 \%$ & $22,20 \%$ \\
\hline & & nde 0 & 52 & \% de fallos & 31,3 & & \\
\hline \multirow{4}{*}{$\begin{array}{c}\text { toma de } \\
\text { decisiones }\end{array}$} & & & & & & & \\
\hline & Vacaciones & nde 1 & 10 & $\%$ & 6,0 & $4,30 \%$ & $4,70 \%$ \\
\hline & & $\pi \mathrm{de} 2$ & 103 & $\%$ & 62,0 & $33,50 \%$ & $25 \%$ \\
\hline & & n de 0 & 53 & $\%$ & 31,9 & & \\
\hline \multirow[t]{3}{*}{ incertidumbre } & Robos & $n$ de 1 & 53 & $\%$ & 31,9 & $28,10 \%$ & $31,30 \%$ \\
\hline & & $n$ de 2 & 56 & $\%$ & 33,7 & $15,40 \%$ & $9,90 \%$ \\
\hline & & n de 0 & 57 & $\%$ & 34,3 & & \\
\hline \multirow{2}{*}{ cambio } & Crecer-1 & $n$ de 1 & 99 & $\%$ de aciertos & 589 & $44.80 \%$ & $36.50 \%$ \\
\hline & & n de 0 & 69 & $\%$ de fallos & 41,1 & & \\
\hline & & & & & & & \\
\hline \multirow{3}{*}{$\begin{array}{l}\text { toma de } \\
\text { decisiones }\end{array}$} & Transporte & n de 1 & 61 & $\%$ & 36,3 & $25,70 \%$ & $22,10 \%$ \\
\hline & & $n \operatorname{de} 2$ & 38 & $\%$ & 22,6 & $11,30 \%$ & $8,80 \%$ \\
\hline & & $n$ de 0 & 69 & $\%$ & 41,1 & & \\
\hline & & & & & & & \\
\hline \multirow[t]{2}{*}{ espacio } & Carpintero & n de 1 & 60 & $\%$ de aciertos & 35,7 & $20 \%$ & $12,90 \%$ \\
\hline & & n de 0 & 108 & \% de fallos & 64,3 & & \\
\hline \multirow[t]{3}{*}{ cantidad } & Vuelo & $n$ de 1 & 31 & $\%$ & 18,7 & $5 / v$ & $5 / \mathrm{V}$ \\
\hline & & $n \operatorname{de} 2$ & 10 & $\%$ & 6,0 & & \\
\hline & & $n$ de 0 & 125 & $\%$ & 75,3 & & \\
\hline
\end{tabular}



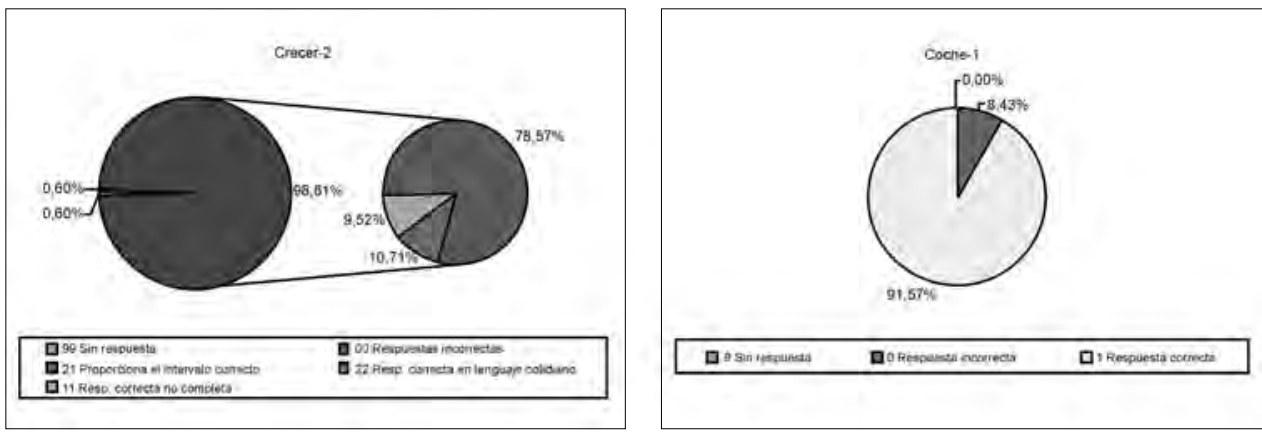

Figura 1
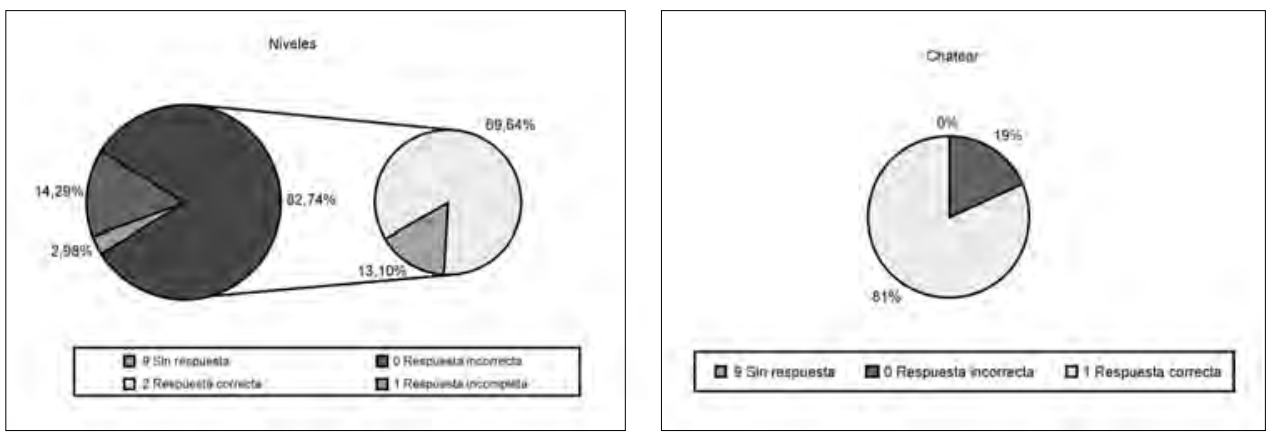

Figura 2
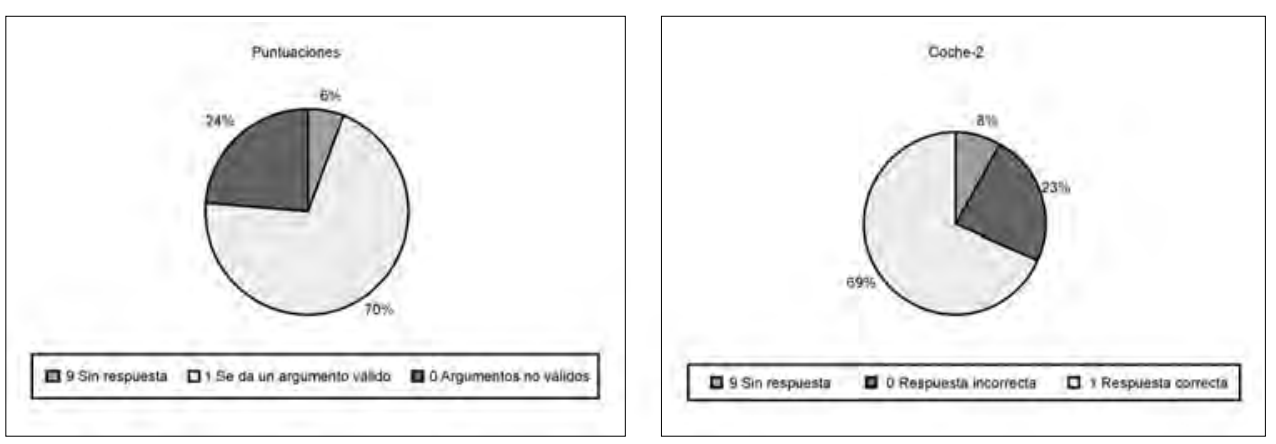

Figura 3 

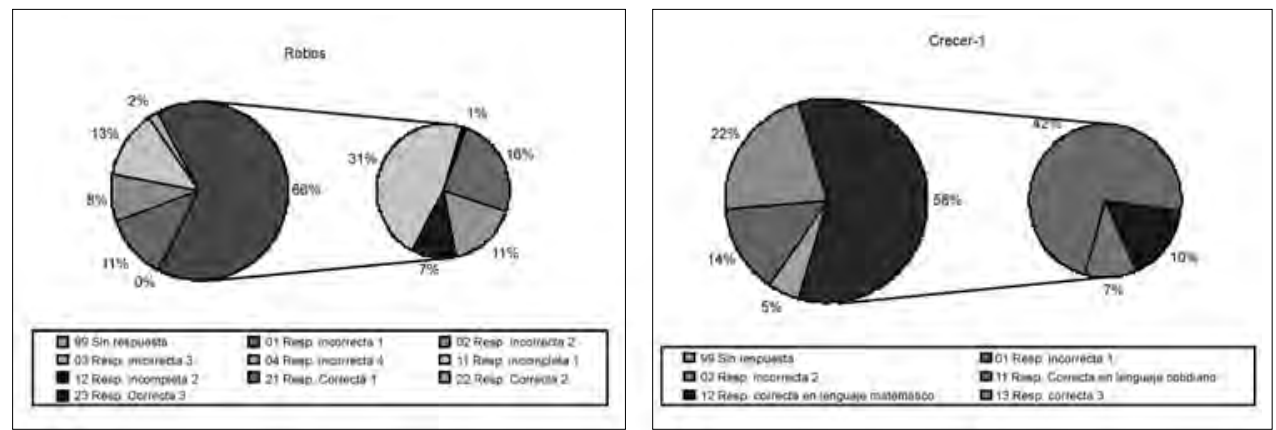

Figura 4
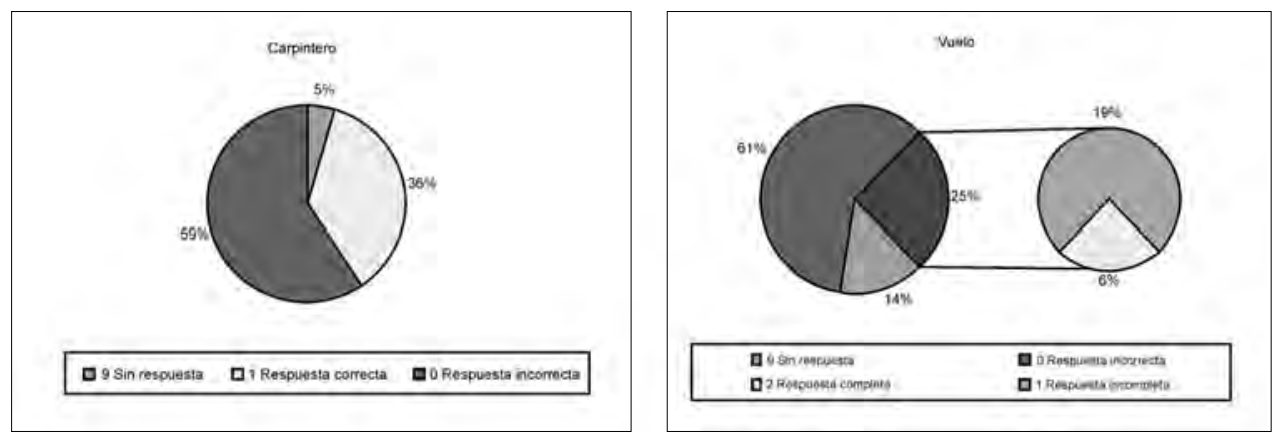

Figura 5
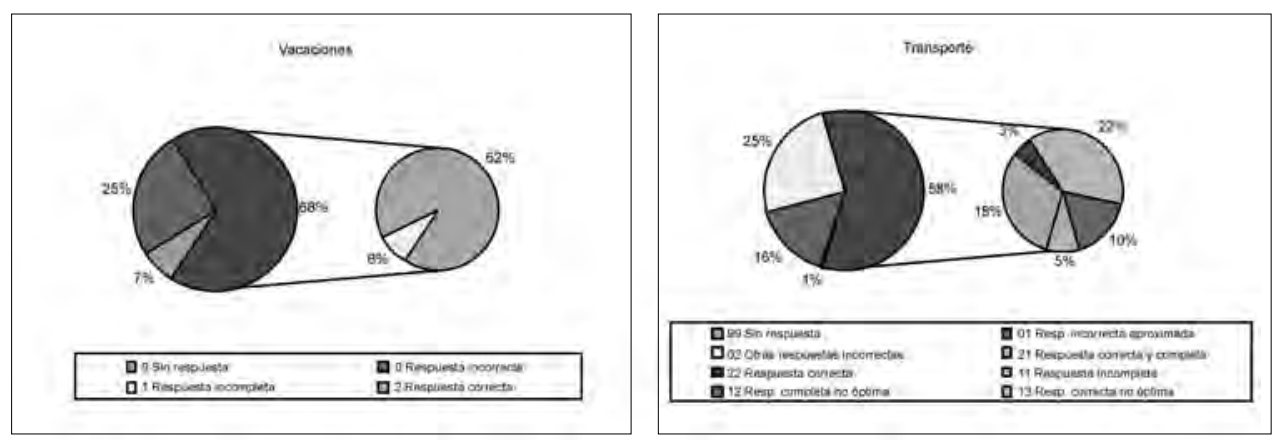

Figura 6 


\subsection{Reflexiones y conclusiones}

En la prueba $B$, nuestros alumnos se muestran relativamente menos competentes en las subáreas de Espacio y forma y Cambio y relaciones (en una situación de cambio no ocurre esto, pero los niveles de dificultad son los más bajos 1 y 3). Se muestran más fuertes en la de Incertidumbre y, sobre todo, en la de Cantidad. Los resultados son muy parecidos a los de las pruebas PISA para el conjunto de los alumnos españoles, aunque los resultados porcentuales de éxito son, obviamente, significativamente superiores. En relación a la Resolución de problemas (toma de decisiones), también se producen resultados porcentuales significativamente superiores.

En la prueba A, la situación parece algo distinta a la de la prueba B; sin embargo el orden de prelación, de acuerdo con los porcentajes de éxito obtenidos, responde a los niveles de dificultad establecidos en PISA con carácter general para cada uno de los ítems. No obstante llama la atención que los peores resultados $(24,70 \%$, puntuación parcial $18,67 \%$, puntuación máxima $6,03 \%)$, corresponden al ítem Vuelo espacial de la subescala Cantidad, que no tiene establecido por PISA nivel de dificultad y para el que sugerimos un nivel máximo. En cuanto a la Resolución de problemas, también los resultados son significativamente superiores tanto en relación a los resultados de España como al conjunto de los países de la OCDE, si bien se sigue manteniendo un alto grado de fracaso.

\section{RELACIONES ENTRE LA COMPETENCIA MATEMÁtiCA DEL FUTURO MAESTRO Y SUS PERFILES EMOCIONALES}

En orden a buscar las mencionadas relaciones hemos trabajado con datos para determinar el perfil emocional matemático de los estudiantes de Magisterio mediante unas escalas que fueron elaboradas y validadas en proyectos I+D anteriores. El estudio, de tipo indagatorio, se realiza sobre una muestra de 332 alumnos pertenecientes a las universidades de La Coruña, Valladolid (campus de Segovia y Valladolid), Zaragoza y Autónoma de Madrid. Las variables que analizamos en esta segunda parte del trabajo son las siguientes:

a) Competencia matemática: el colectivo considerado está formado por los alumnos que realizaron la prueba sobre competencia matemática denominada cuestionario B. Esta prueba planteaba una cuestión del ámbito de la aritmética y otra del ámbito de la geometría, situación que no se daba en el cuestionario A. Los resultados globales sobre la competencia matemática que muestran los 332 estudiantes se muestran en la Tabla 3. A los efectos de un análisis detallado de los datos agruparemos el nivel de competencia matemática en tres niveles: alto, medio y bajo, según la Tabla 4.

b) Creencias sobre las matemáticas: Se considera la información proporcionada por los alumnos encuestados referida a los ítems sobre dificultad, utilidad y agrado, tanto en aspectos conceptuales como procedimentales, sobre los dos tópicos más importantes del currículo de educación primaria: aritmética y geometría.

c) Creencia sobre el desempeño profesional: Se consideran dos ítems referentes al trabajo como profesores de matemáticas en Educación Primaria. El primer 
ítem se refiere a la capacitación para entender las claves de la enseñanza de las matemáticas, mientras que el segundo se refiere a las creencias de los encuestados sobre la seguridad que tienen al explicar las matemáticas.

\section{Tabla 3}

\begin{tabular}{|c|c|c|}
\hline Calificación & Número de estudiantes & Porcentaje \\
\hline 10 & 15 & 4,5 \\
\hline 8,9 & 30 & 9 \\
\hline 7,8 & 59 & 17,8 \\
\hline 6,7 & 67 & 20,3 \\
\hline 5,6 & 63 & 18,9 \\
\hline 4,4 & 42 & 12,7 \\
\hline 3,3 & 30 & 9 \\
\hline 2,2 & 19 & 5,7 \\
\hline 1,1 & 6 & 1,8 \\
\hline 0 & 1 & 0,3 \\
\hline Total & 332 & 100 \\
\hline
\end{tabular}

\section{Tabla 4}

\begin{tabular}{|c|c|c|c|}
\hline Nivel de competencia matemática & Calificación & $\mathbf{N}^{\mathbf{0}}$ de estudiantes & Porcentaje \\
\hline Alto & Entre 7,8 y 10 & 104 & 31,3 \\
\hline Medio & Entre 5,6 y 6,7 & 130 & 39,2 \\
\hline Bajo & Entre 0 y 4,4 & 98 & 29,5 \\
\hline & Total & 332 & 100 \\
\hline
\end{tabular}

\subsection{Resultados}

Se establecen los siguientes resultados:

EN LAS RELACIONES ENTRE COMPETENCIA MATEMÁTICA Y DIFICULTAD DE LAS MATEMÁTICAS

3.1.1. El gráfico de la Figura 7 muestra los resultados obtenidos al relacionar la competencia matemática de los alumnos y la dificultad que asocian a los conceptos aritméticos. Como puede observarse en el gráfico la competencia matemática de los futuros maestros está relacionado con las dificultades que asocian a los aspectos conceptuales de la aritmética, de modo que a mayor nivel de competencia matemática menor es la percepción de dificultades en los conceptos aritméticos. 


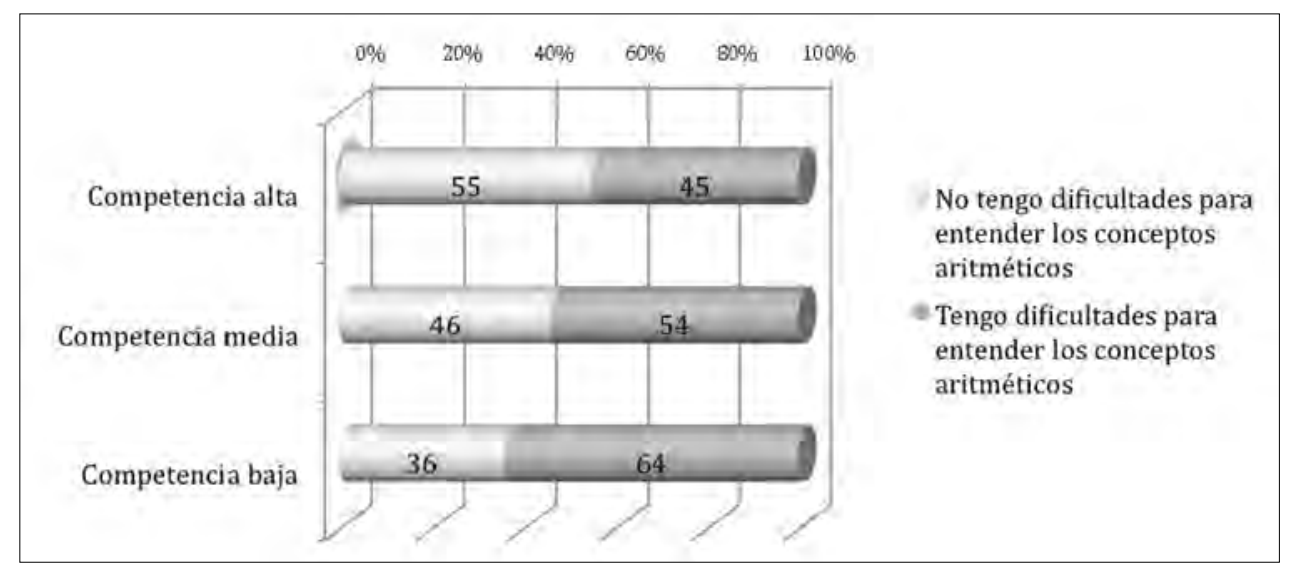

Figura 7

3.1.2. La Figura 8 muestra los resultados obtenidos al relacionar la competencia matemática de los alumnos y la dificultad que asocian a los conceptos geométricos. El gráfico muestra que el rendimiento matemático de los futuros maestros está relacionado con las dificultades que asocian a los aspectos conceptuales de la geometría. Los resultados son parecidos a los del apartado 3.1.1, aunque con ligeras variaciones: los alumnos de competencia matemática alta tienen menos dificultades con los conceptos aritméticos que con los conceptos geométricos, mientras que los alumnos de competencia matemática baja tienen más dificultades con los conceptos geométricos que con los conceptos aritméticos.

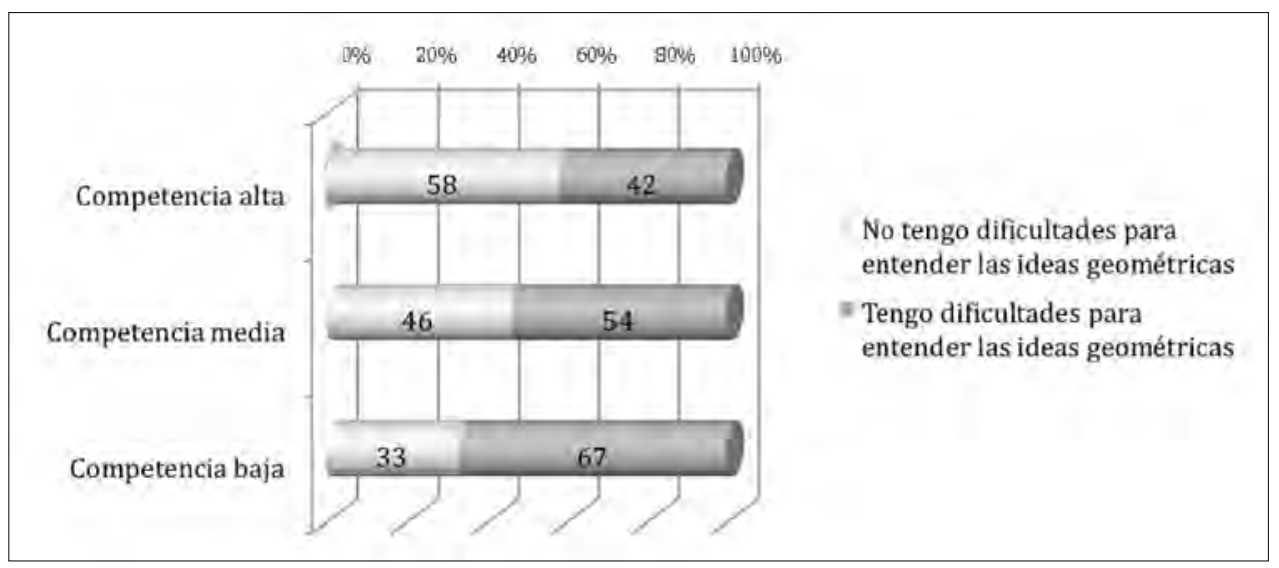

Figura 8

3.1.3. El gráfico de la Figura 9 muestra los resultados obtenidos al relacionar la competencia matemática de los alumnos y la dificultad que asocian a los procedimientos aritméticos. El gráfico nos ofrece dos informaciones que consideramos rele- 
vantes. De una parte, los futuros maestros declaran, de forma mayoritaria, no tener dificultades con los procedimientos aritméticos, aun cuando los alumnos de competencia matemática baja tienen más dificultades que los alumnos de competencia matemática media y alta. De otra parte, al comparar estos resultados con los del apartado 3.1.1 se observa que los estudiantes para Maestro tiene más dificultades con los conceptos aritméticos que con los procedimientos aritméticos.

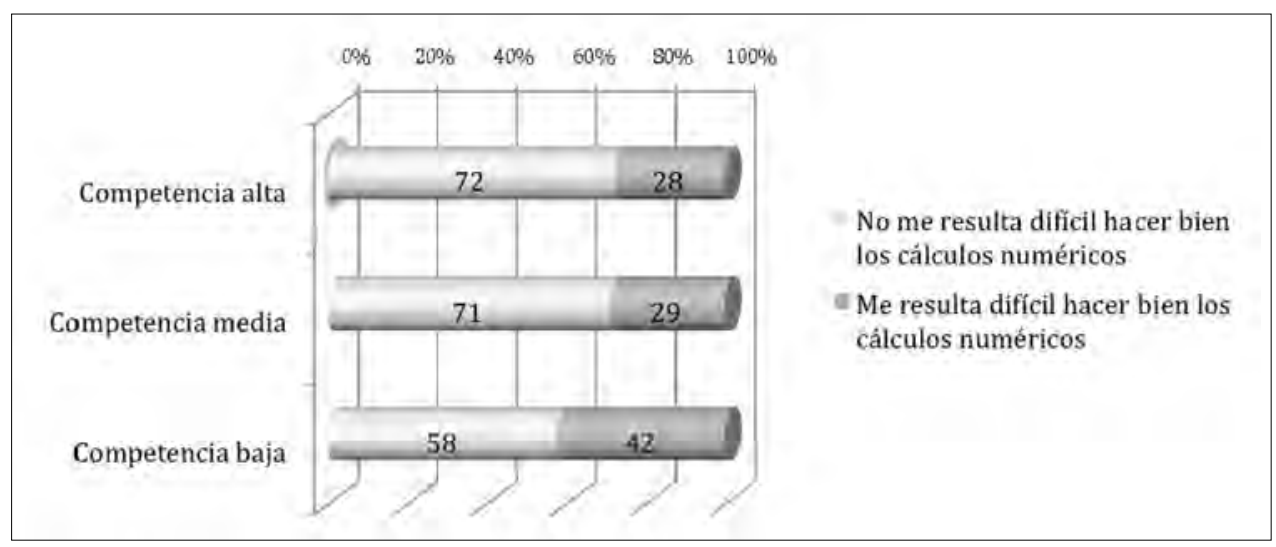

Figura 9

3.1.4. Al relacionar la competencia matemática de los alumnos y la dificultad que asocian a los procedimientos geométricos, se obtienen los resultados recogidos en la Figura 10. El gráfico nos indica que los futuros maestros establecen una relación entre su competencia matemática y las dificultades que encuentran con los procedimientos geométricos. Además, al comparar estos resultados con los del apartado 1.2. se observa que hay diferencias muy pequeñas entre las dificultades de los conceptos geométricos y los procedimientos geométricos.

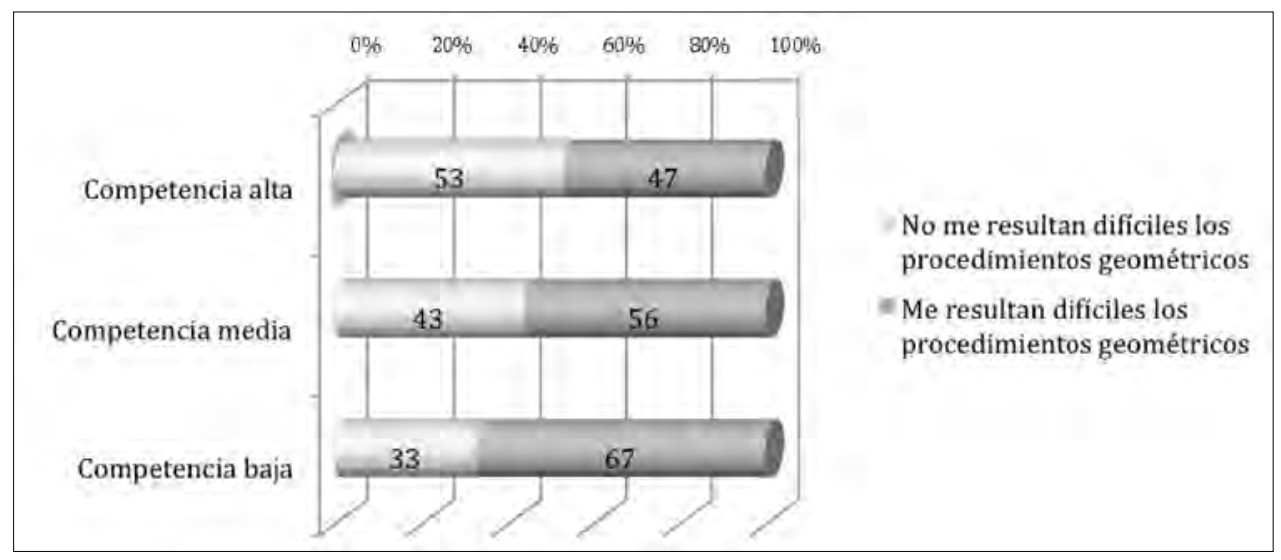

Figura 10 
3.1.5. Al considerar conjuntamente los cuatro ítems analizados en los apartados 3.1.1, 3.1.2, 3.1.3 y 3.1.4, se observa una relación entre la competencia matemática de los alumnos y los valores medios que obtienen en los cuatro ítems, tal y como queda resumido en la Tabla 5. Estos resultados ponen de manifiesto que existe una estrecha relación entre el grado de competencia matemática de los estudiantes para Maestro y la dificultades que dicen poseer en relación a conceptos y procedimientos matemáticos. Así, los estudiantes que muestran una competencia matemática más baja admiten tener mayores dificultades en conceptos y procedimientos aritméticos y geométricos y, al revés, los estudiantes de mayor competencia matemática perciben las matemáticas como menos difíciles. Estas relaciones son similares a las obtenidas en estudios recientes sobre relaciones entre competencia matemática y factores emocionales (Sáenz, 2007; Penalva et al, 2009). Además, se observa una tendencia generalizada a considerar que la geometría presenta mayores dificultades que la aritmética. Y también se considera que el conocimiento conceptual, tanto aritmético como geométrico, presenta mayores dificultades que el conocimiento procedimental. Estos resultados indican que las ideas sobre dificultades de las matemáticas de los alumnos no universitarios (Corbalán et al, 1984; Hidalgo et al, 2000) siguen con resultados similares en los estudiantes universitarios del grado de Maestro en Educación Primaria. Entre las relaciones estudiadas cabe destacar el hecho de que haya 56 estudiantes del grupo de competencia baja que dicen no tener de dificultades con los contenidos matemáticos. Traducidos estos datos a toda la muestra cabe decir que casi el $20 \%$ de los estudiantes para Maestro tienen una competencia matemática baja y no son conscientes de las limitaciones de su propio conocimiento matemático, pues afirman no tener dificultades con la aritmética ni con la geometría.

\section{Tabla 5}

\begin{tabular}{|c|c|c|c|}
\hline & Competencia alta & Competencia media & Competencia baja \\
\hline Media & 1,4 & 1,6 & 1,7 \\
\hline
\end{tabular}

EN LAS RELACIONES ENTRE COMPETENCIA MATEMÁTICA Y AGRADO ANTE LAS MATEMÁTICAS

3.2.1. El gráfico de la Figura $\mathbf{1 1}$ muestra los resultados obtenidos al relacionar la competencia matemática de los alumnos y el gusto que asocian al trabajo en aritmética. Los estudiantes de Magisterio afirman que les gusta utilizar la aritmética aunque esta pauta afectiva es más acusada en los estudiantes de competencia matemática alta. Y cabe destacar que entre los estudiantes de competencia matemática media y baja el sentimiento mayoritario es el de no gustarles la utilización de la aritmética, situación que nos parece preocupante porque los conocimientos aritméticos están presentes en la mayor parte de las actividades matemáticas de los ciudadanos. 


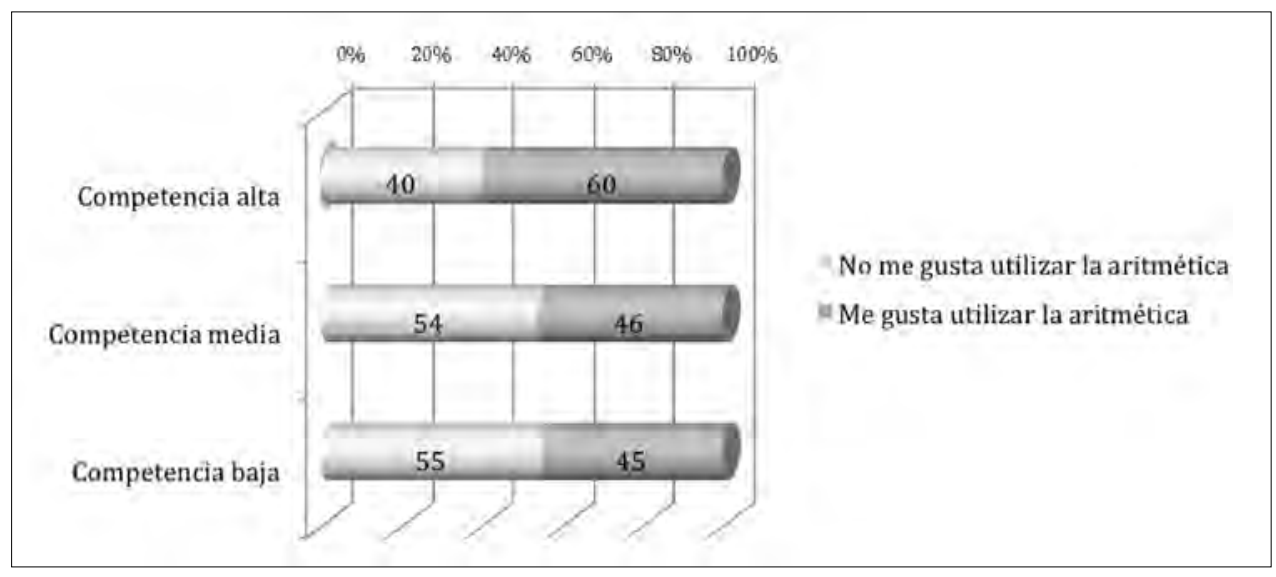

Figura 11

3.2.2. La Figura 12 muestra los resultados obtenidos al relacionar la competencia matemática de los alumnos y el gusto que asocian al trabajo en geometría. Se observa que los estudiantes de mayor competencia matemática muestran más agrado por la geometría y que el agrado por ésta disciplina va disminuyendo en los estudiantes que muestran menor competencia matemática.

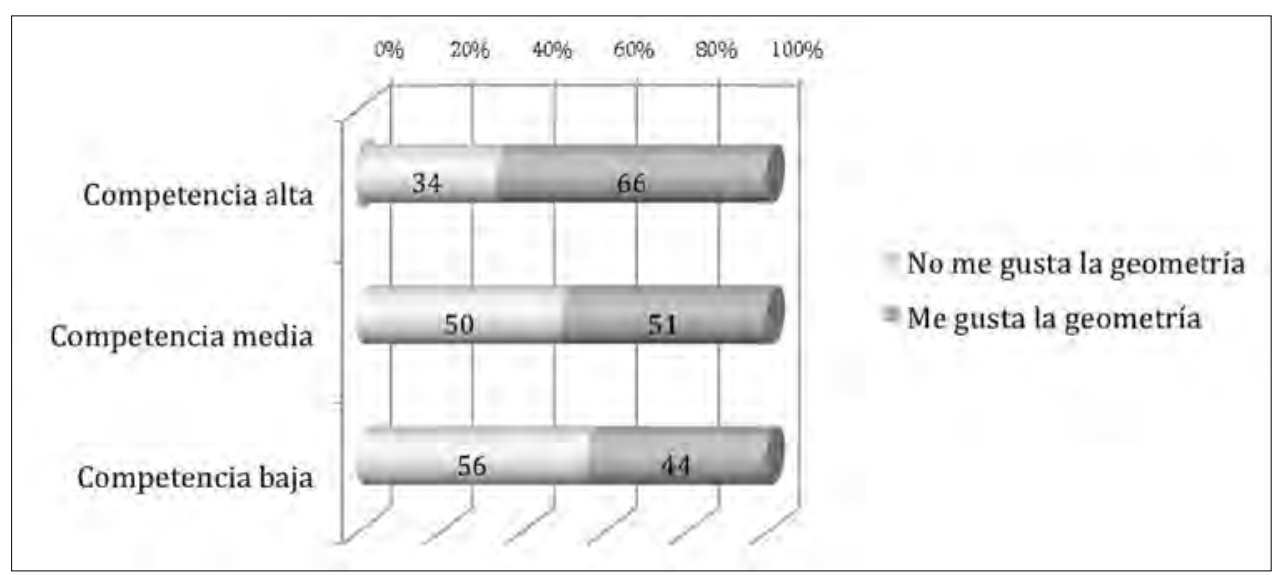

Figura 12

3.2.3. El gráfico de la Figura $\mathbf{1 3}$ muestra los resultados obtenidos al relacionar la competencia matemática de los alumnos y el agrado que asocian al trabajo procedimental en aritmética. Este ítem, al igual que los otros anteriormente estudiados de la variable agrado, sigue la misma pauta de comportamiento entre los grupos de diferente competencia matemática: los de mayor competencia muestran agrado mientras que esta percepción disminuye en los grupos de competencia matemática media y baja. 


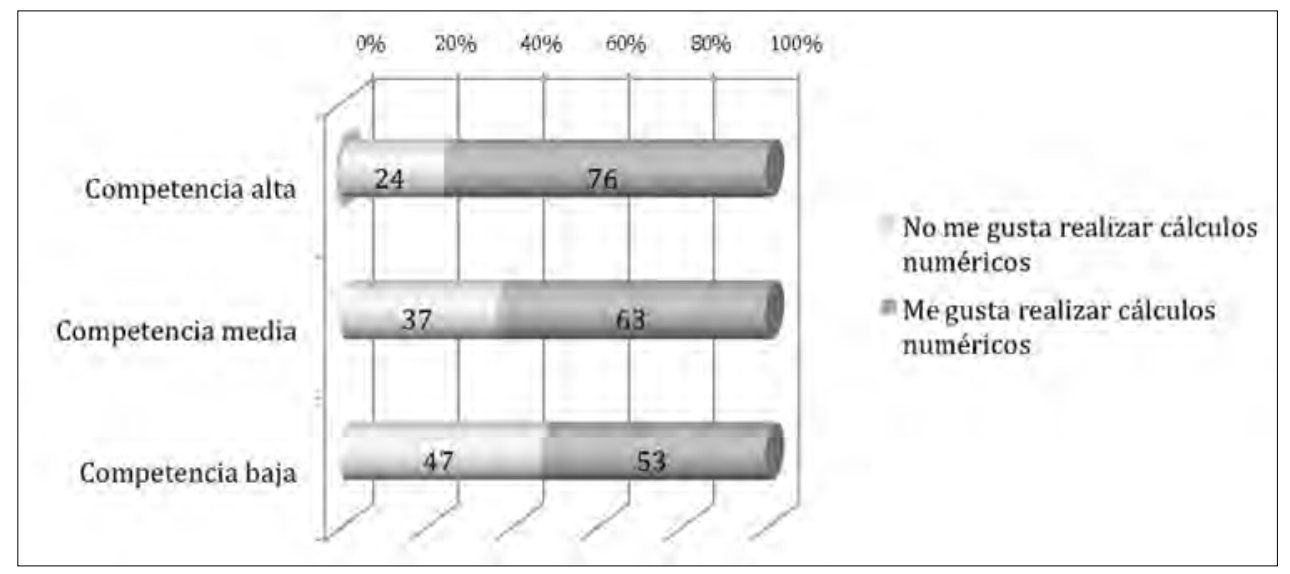

Figura 13

3.2.4. La Figura $\mathbf{1 4}$ muestra los resultados obtenidos al relacionar la competencia matemática de los alumnos y el agrado que asocian al trabajo procedimental en geometría. Se observa un comportamiento análogo de los estudiantes en los dos ítem relacionados con la geometría: tanto en procedimientos como en conceptos, los alumnos de mayor competencia matemática muestran mayor agrado en la aplicación de conceptos y procedimientos. Una característica distintiva entre los conceptos y procedimientos radica en que la aplicación de éstos últimos gusta menos que la utilización de conceptos geométricos.

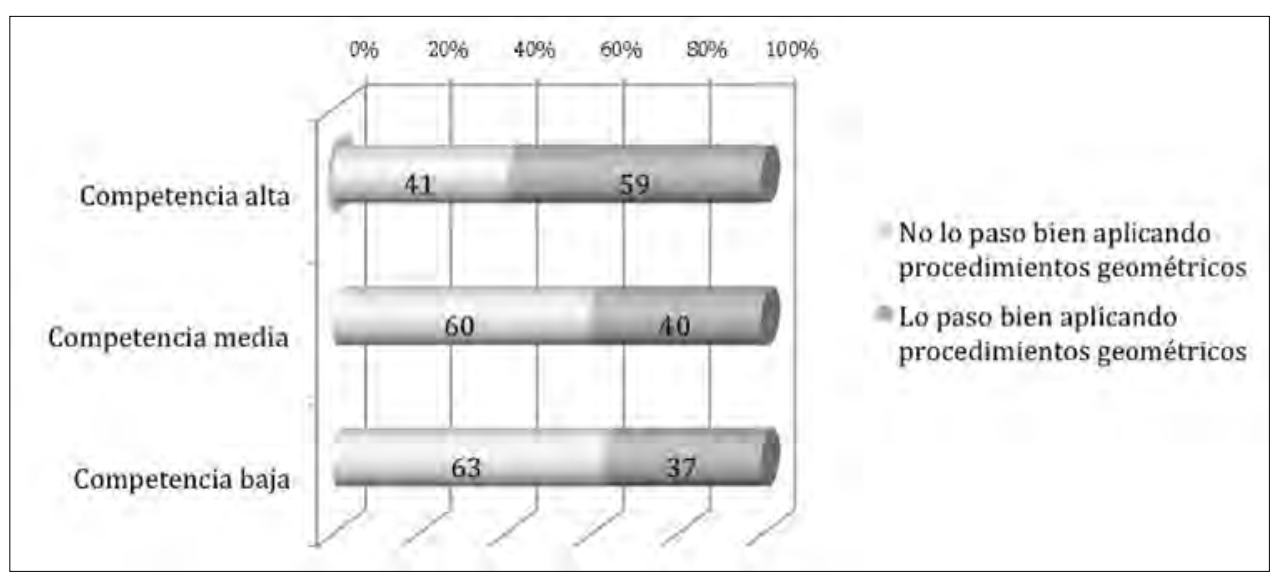

Figura 14

3.2.5. La realización de cálculos numéricos es la actividad matemática preferida por los estudiantes de Magisterio frente a la realización de procedimientos geométricos o la de utilización de conceptos aritméticos y geométricos. No resulta sorprendente la constatación de la preferencia por la realización de cálculos numéricos 
dado que se trata de una actividad de fuerte implantación en la escuela que hace que los estudiantes de Magisterio la vinculen con la enseñanza de las matemáticas en Educación Primaria. Resulta Ilamativo que los estudiantes consideren, por una parte, que el trabajo en geometría les gusta más que el trabajo en aritmética y que, por otra parte, consideren sentirse más a gusto utilizando procedimientos aritméticos que geométricos. Una explicación plausible puede encontrarse en su percepción de que la geometría tiene una componente estética más acusada que la aritmética, mientras que los procedimientos geométricos (varias definiciones para una misma figura, clasificación inclusiva o exclusiva de figuras, construcción de figuras y de lugares geométricos, etc.) les resultan más complejos que los procedimientos aritméticos (se utiliza una única definición de cada concepto, las clasificaciones son exclusivas y poco utilizadas, no hay construcciones, etc.).

\section{EN LAS RELACIONES ENTRE COMPETENCIA MATEMÁTICA E IMPORTANCIA DE LAS MATEMÁTICAS}

3.3.1. El gráfico de la Figura 15 muestra los resultados obtenidos al relacionar la competencia matemática de los alumnos y la importancia que asocian a la aritmética. El gráfico muestra con claridad que para los estudiantes de Magisterio los números resultan muy importantes para la vida adulta. También muestra con claridad que la importancia de los números no depende significativamente de la competencia matemática de estos estudiantes.

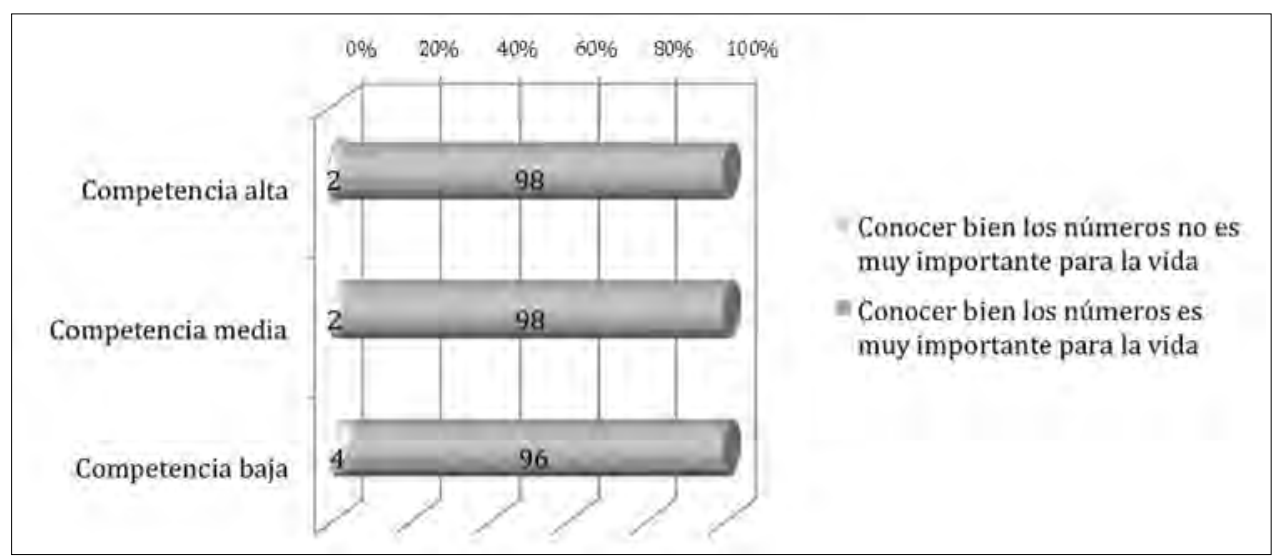

Figura 15

3.3.2. La Figura 16 muestra los resultados obtenidos al relacionar la competencia matemática de los alumnos y la importancia que asocian a la geometría. Se observa que los estudiantes conceden a la geometría una gran importancia para la vida, aunque tal importancia decrece al decrecer la competencia matemática de dichos estudiantes. Es Ilamativo que casi la tercera parte de los estudiantes con competencia media o baja digan que la geometría no tiene importancia para la vida; posiblemente porque no asocien la geometría escolar con situaciones problemáticas 
relacionadas con actividades de diseño, de distribución de espacios, de elaboración de maquetas, de localización, etc.

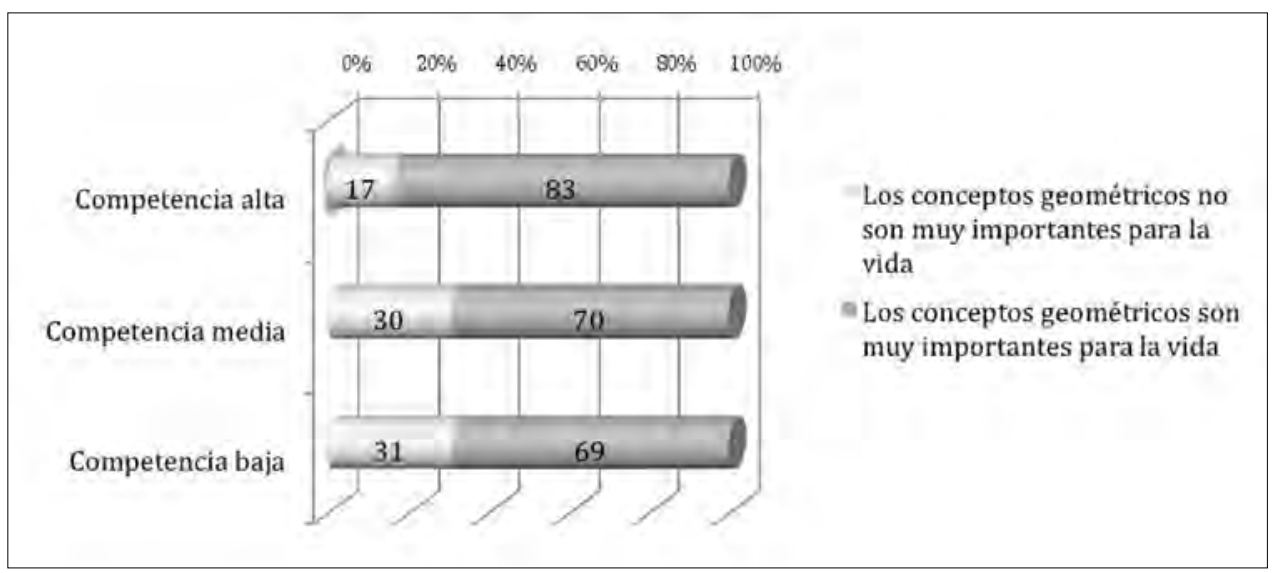

Figura 16

3.3.3. El gráfico de la Figura $\mathbf{1 7}$ muestra los resultados obtenidos al relacionar la competencia matemática de los alumnos y la importancia que asocian a los procedimientos aritméticos. Estos resultados ponen de relieve que para los estudiantes de Magisterio los cálculos numéricos tienen una presencia muy destacada en las actividades cotidianas. Aunque resulta llamativo que la quinta parte de los estudiantes de competencia matemática media y la quinta parte de los estudiantes de competencia matemática baja coincidan en considerar que los cálculos numéricos no son muy importantes para la vida.

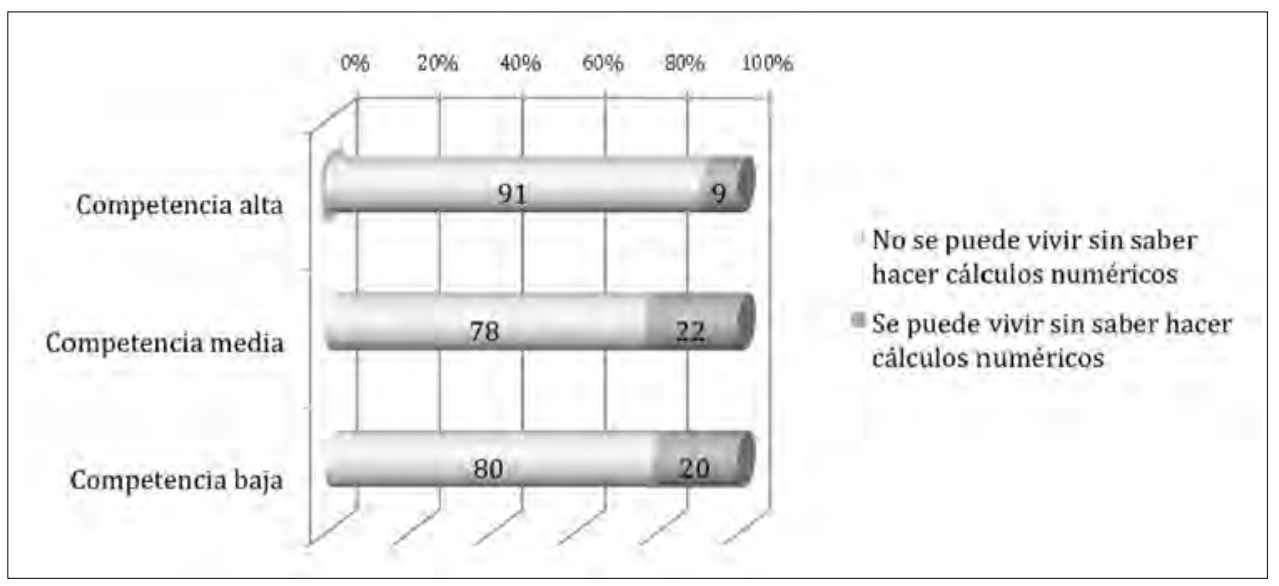

Figura 17 
3.3.4. La Figura 18 muestra los resultados obtenidos al relacionar la competencia matemática de los alumnos y la importancia que asocian a los procedimientos geométricos. Los conocimientos procedimentales de la geometría se consideran de gran importancia para la vida, aunque esa importancia va disminuyendo al disminuir la competencia matemática de los estudiantes.

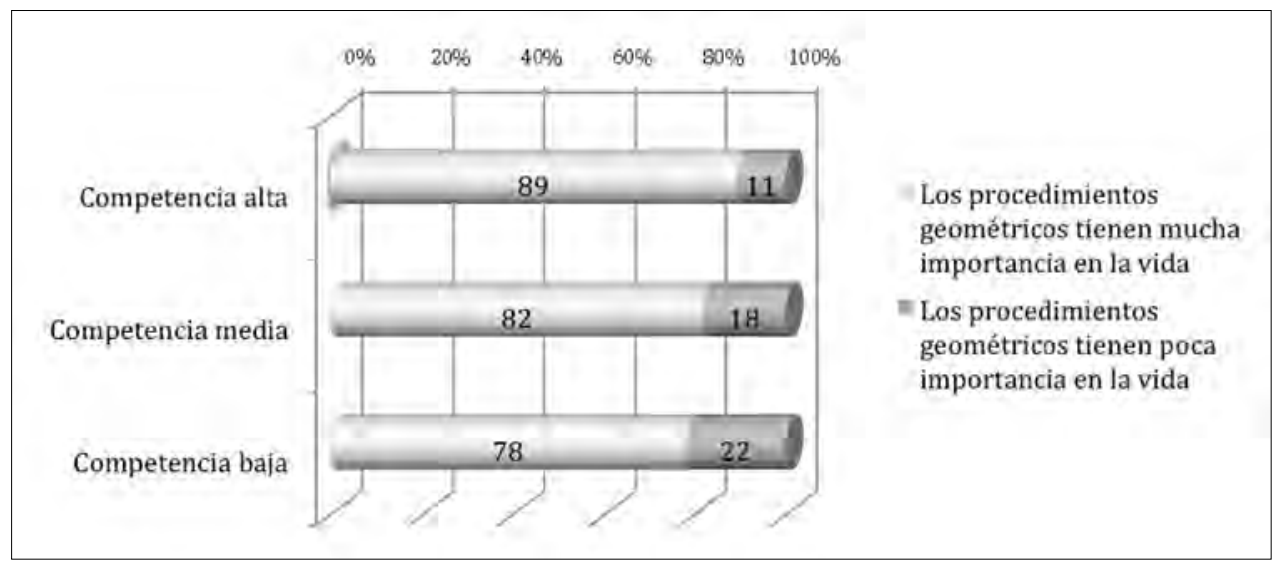

Figura 18

3.3.5. Para casi la totalidad de los estudiantes de Magisterio encuestados las ideas aritméticas son importantes en la vida; mientras que las ideas geométricas no son importantes para casi la tercera parte de dichos alumnos. Entendemos que para una tercera parte de los estudiantes las ideas aritmética hay que usarlas en la vida adulta, mientras que las ideas geométricas son prescindibles. En cuanto a los procedimientos, tanto aritméticos como geométricos, se les concede una gran importancia en la vida, aunque la competencia matemática está relacionada con el grado de importancia que conceden los estudiantes al conocimiento procedimental.

\section{EN LAS RELACIONES ENTRE COMPETENCIA MATEMÁTICA Y DESEMPEÑO PROFESIONAL}

3.4.1. El gráfico de la Figura 19 muestra los resultados obtenidos al relacionar la competencia matemática de los alumnos y su capacidad para entender las claves de la enseñanza de las matemáticas. Como puede constatarse en el gráfico, la gran mayoría de los estudiantes para Maestro se muestran confiados en que pueden entender las claves de la enseñanza de las matemáticas y, por lo tanto, en cumplir con eficacia su desempeño profesional. Llama la atención este alto grado de confianza en el desarrollo profesional en alumnos que inician sus estudios universitarios; y también resulta sorprendente que el $89 \%$ de los estudiantes con rendimiento bajo en matemáticas se consideren capacitados para entender las claves de la enseñanza de las matemáticas. Nos encontramos, por tanto, que los futuros profesores consideran que la enseñanza de las matemáticas depende del conocimiento del método de enseñanza más que de su propio conocimiento de las matemáticas esco- 
lares. Estas creencias podrían tener su origen en la formación psicopedagógica que reciben los estudiantes para Maestro en el primer curso de los estudios de grado, o en la idea forjada en su experiencia como alumnos de que la profesión docente se sustenta en conocimientos y aptitudes entre las que no se incluye el conocimiento de la disciplina objeto de la enseñanza. Los estudiantes conciben que la labor del docente viene determinada por la correcta aplicación de un método de enseñanza; es decir, conciben a los Maestros como técnicos docentes. Estas creencias de los estudiantes a buen seguro que se contraponen con las creencias de sus formadores que consideran a los docentes como tecnólogos: los Maestros deben conocer la teoría que sustenta el método de enseñanza.

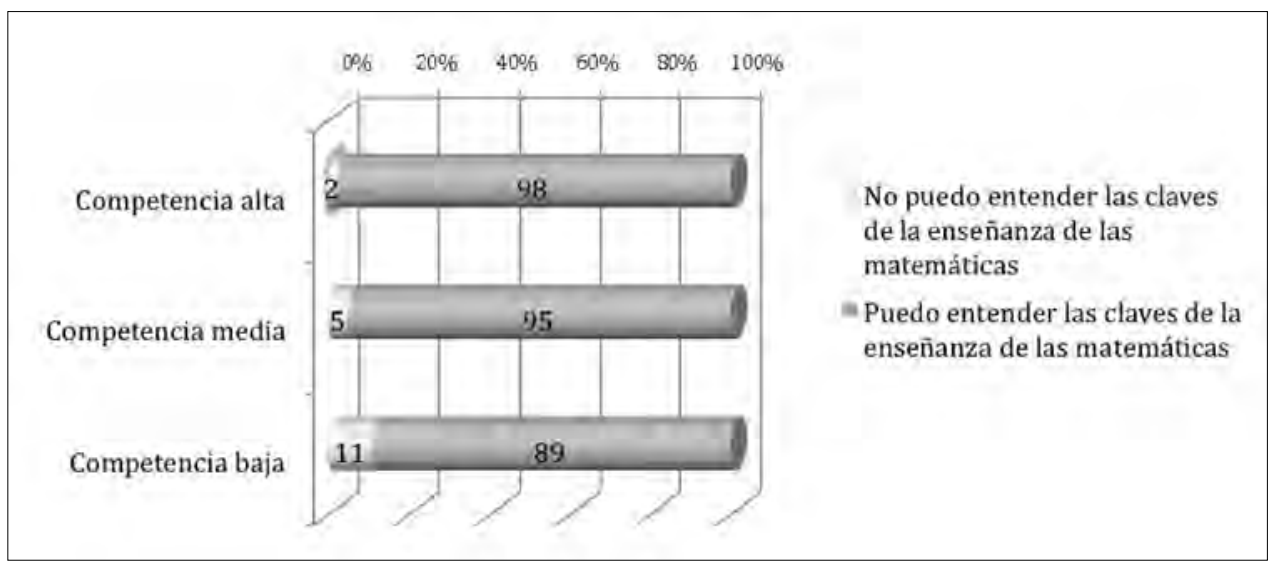

Figura 19

3.4.2. Los resultados obtenidos al relacionar la competencia matemática de los alumnos y la seguridad que tienen para enseñar matemáticas, se muestran en la Figura 20. De los datos recogidos en el gráfico se deduce la existencia de una relación entre el nivel de competencia matemática y el factor inseguridad: los estudiantes de menor competencia matemática dicen sentirse inseguros en un porcentaje muy superior al de los estudiantes de competencia matemática media y alta. Al observar los resultados del factor inseguridad en el grupo de competencia matemática baja detectamos casi el $40 \%$ de los estudiante niegan mostrarse inseguros al explicar matemáticas. Con los datos de que disponemos hasta este momento no podemos pronunciarnos sobre si la percepción de estos estudiantes es fruto de la reflexión sobre su competencia profesional o si es fruto de experiencias previas como profesores. Se trata de una cuestión que merecería ser objeto de un futuro trabajo de investigación. 


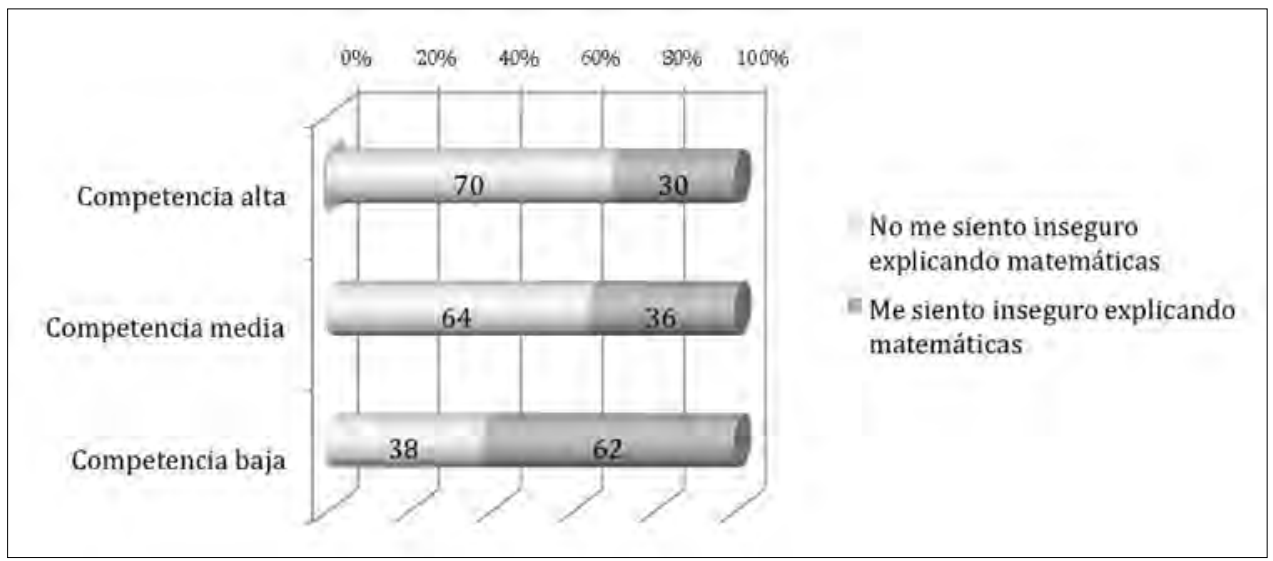

Figura 20

\section{Competencia matemática, Creencias sobre la naturaleza de las matemáticas Y CREENCIAS SOBRE EL DESARROLLO PROFESIONAL}

4.1. Al relacionar el indicador dificultad de las matemáticas con la capacidad de entender las claves de la enseñanza de las matemáticas se obtienen los resultados de la Tabla 6. Según estos resultados el estudiante que comienza los estudios de Magisterio tiene una visión ingenua de la enseñanza de las matemáticas escolares sustentada en la idea de que "como considero que las matemáticas no son difíciles tampoco tendré dificultades para entender las claves de la enseñanza de las matemáticas" Al establecer relaciones entre las tres variables: competencia matemática, dificultad de las matemáticas y capacidad para entender las claves de la enseñanza de las matemáticas, nos ha parecido destacable comentar las respuestas de los 42 estudiantes de competencia matemática baja y que admiten tener dificultades con los contendidos matemáticos escolares. El gráfico de la Figura 21 contiene la información correspondiente. Observamos el grupo de 33 estudiantes que afirman su capacidad para entender las claves de la enseñanza de las matemáticas. Estos estudiantes se sienten bien cualificados profesionalmente a pesar de tener una competencia matemática baja y admitir dificultades con las matemáticas. Tan solo 9 estudiantes de los 42 se cuestionan su valía profesional al ser conscientes de las dificultades que tienen en matemáticas. Téngase en cuenta que este grupo de 33 alumnos representa el 10\% del total de la muestra, porcentaje que pensamos podría aumentar al adaptar la prueba de competencia matemática al nivel universitario. Este resultado lo consideramos relevante puesto que detecta la presencia de un grupo importante de alumnos con un grado bajo de competencia matemática, que admiten tener dificultades con las matemáticas y que, sorprendentemente, se siente capaces o muy capaces de entender las claves de la enseñanza de las matemáticas. 
Tabla 6

\begin{tabular}{|l|c|c|c|}
\hline & $\begin{array}{c}\text { Competencia } \\
\text { alta }\end{array}$ & $\begin{array}{c}\text { Competencia } \\
\text { media }\end{array}$ & $\begin{array}{c}\text { Competencia } \\
\text { baja }\end{array}$ \\
\hline Media del ítem sobre Dificultad & 1,4 & 1,6 & 1,8 \\
\hline Media del ítem Entender las claves & 2,9 & 2,6 & 2,5 \\
\hline
\end{tabular}

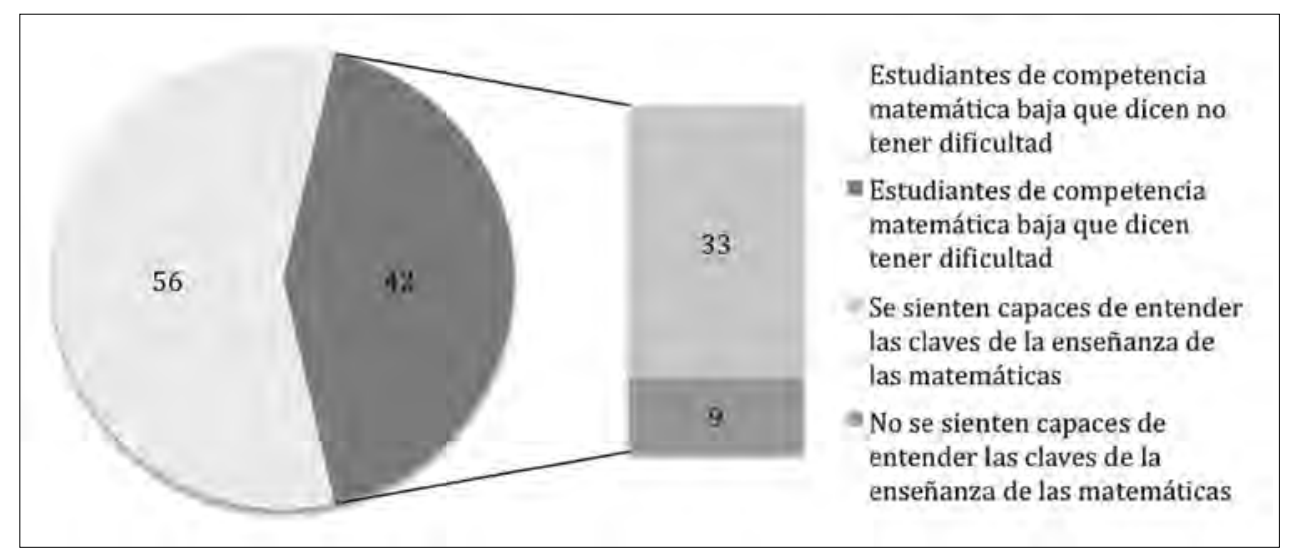

Figura 21

4.2. Al estudiar el indicador dificultad de las matemáticas con la seguridad en tareas docentes se obtienen los resultados reflejados en la Tabla 7. Cabe mencionar que en el grupo de estudiantes de competencia matemática baja la media de la variable Seguridad se acerca a 2 puntos, lo que indica que estos estudiantes están cercanos a admitir que se sienten inseguros explicando matemáticas, mientras que los alumnos de competencia matemática alta se sienten más seguros al explicar matemáticas. Analizando las relaciones entre las tres variables competencia matemática, dificultad de las matemáticas y seguridad al explicar matemáticas, nos ha parecido destacable comentar las respuestas de los 56 estudiantes del grupo de competencia matemática baja y que admiten no tener de dificultades con los contendidos matemáticos escolares. El gráfico de la Figura 22 contiene la información correspondiente. Especialmente significativo es el resultado obtenido por los 30 estudiantes señalados en el gráfico. Estos estudiantes, que representan aproximadamente el $10 \%$ del total de la muestra, son estudiantes de rendimiento bajo en competencia matemática y que afirman no sentirse inseguros explicando matemáticas. De nuevo constatamos relaciones no esperables entre las variables competencia matemática y competencia profesional en el grupo de estudiantes de competencia matemática baja. Cabe conjeturar que el origen de esta situación es achacable, de una parte, a la percepción que poseen estos estudiantes de Magisterio sobre su propia competencia matemática y, de otra parte, un cierto desconocimiento de la génesis de las matemáticas escolares y de las 
exigencias requeridas para el correcto desempeño profesional sobre la enseñanza de las matemáticas.

Tabla 7

\begin{tabular}{|l|c|c|c|}
\hline & $\begin{array}{c}\text { Competencia } \\
\text { alta }\end{array}$ & $\begin{array}{c}\text { Competencia } \\
\text { media }\end{array}$ & $\begin{array}{c}\text { Competencia } \\
\text { baja }\end{array}$ \\
\hline Media del ítem sobre Dificultad & 1,4 & 1,6 & 1,8 \\
\hline Media del ítem sobre Seguridad & 1,3 & 1,5 & 1,9 \\
\hline
\end{tabular}

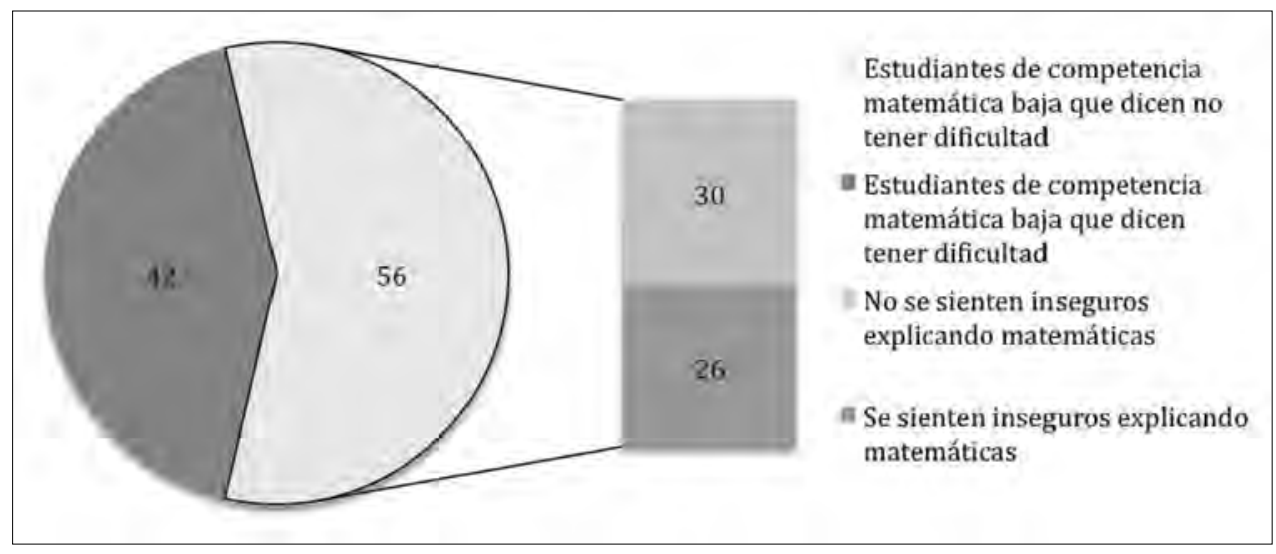

Figura 22

\section{REFLEXIONES Y CONCLUSIONES}

1. Los estudiantes de Magisterio ponen de manifiesto que la aritmética y la geometría tienen gran importancia en la vida adulta; es decir, reconocen que los dos núcleos con mayor presencia en el currículo escolar son importantes en la formación matemática de los ciudadanos. Sin embargo, es de destacar que para una tercera parte de los estudiantes las ideas aritméticas hay que usarlas en la vida adulta, mientras que las ideas geométricas son prescindibles.

2. Una cantidad algo superior a la mitad de los estudiantes para Maestro, el 52\%, reconoce que tiene dificultades para entender los aspectos conceptuales tanto aritméticos como geométricos. Y es de destacar que para el 57\% de dichos estudiantes los procedimientos geométricos les resultan difíciles, posiblemente porque están menos adiestrados en los métodos y en las herramientas propias de la geometría.

3. Los estudiantes encuestados no proporcionan datos relevantes sobre el agrado que sienten al estudiar los procedimientos aritméticos; pero sí resulta preocu- 
pante que el $55 \%$ de la totalidad de la muestra indica que no le agrada el trabajo procedimental en geometría. Entendemos, por tanto, que los estudiantes se encuentran más a gusto cuando realizan cálculos numéricos que cuando tienen que hacer tareas de clasificación o representación de figuras geométricas.

4. Los estudiantes encuestados manifiestan que les resulta más difícil entender los conceptos geométricos que los aritméticos; además sienten un nivel más alto de agrado hacia la aritmética que hacia la geometría. Esta situación entendemos que surge como reflejo de una práctica educativa que prioriza los contenidos aritméticos sobre los geométricos y que dedica la mayor parte del trabajo, tanto docente como discente, a la práctica de destrezas (Gairín, J.M. y Muñoz, J.M., 2005). Estos resultados deben orientar la labor de los formadores de los futuros Maestros en el sentido de modificar las creencias de los estudiantes sobre la naturaleza de las matemáticas y, en consecuencia, las creencias de dichos estudiantes sobre las pecualiaridades de su enseñanza y de su aprendizaje; de lo contrario estos futuros Maestros tenderán a reproducir el modelo en el que aprendieron.

5. La competencia matemática puede relacionarse de forma inversa con la dificultad que encuentran los futuros Maestros en los conceptos y procedimientos tanto aritméticos como geométricos: cuanto mayor es la competencia matemática menor es la dificultad de comprensión de las matemáticas, y cuanto menor es la competencia matemática mayor es la dificultad de comprensión de las matemáticas.

6. La competencia matemática puede relacionarse de forma directa con el agrado que se experimenta al realizar tareas matemáticas: cuanto mayor es la competencia matemática mayor es el agrado de hacer matemáticas, y cuanto menor es la competencia matemática menor es el agrado de hacer matemáticas.

7. Con independencia de su competencia profesional los estudiantes de Magisterio se consideran capacitados para cumplir con eficacia las labores porque se sienten capaces de entender las claves del proceso de enseñanza y aprendizaje de las matemáticas.

8. Se ha puesto de manifiesto una relación directa entre la competencia matemática de los estudiantes encuestados y la seguridad que manifiestan para enseñar matemáticas: los alumnos menos competentes se sienten más inseguros que los alumnos más competentes

9. En esta investigación se ha constatado que la competencia matemática de los estudiantes para Maestro se puede considerar como elemento vehiculador de sus creencias sobre la naturaleza de las matemáticas y sobre sus creencias acerca del desarrollo profesional del docente.

Convendría, por tanto, disponer de una prueba sobre competencia matemática fiable adaptada tanto al nivel universitario de los estudiantes de Magisterio, como a los objetivos de su formación profesional. Con la ayuda de esta prueba, el formador de los futuros Maestros dispondría de datos suficientes para delimitar el perfil emocional y de expectativas profesionales de sus alumnos, lo que sin duda, facilitaría el diseño del correspondiente plan de formación. 
PERFIL EMOCIONAL Y COMPETENCIAS MATEMÁTICAS DE LOS ESTUDIANTES DEL GRADO DE EDUCACIÓN...

\section{REFERENCIAS BIBLIOGRÁFICAS}

ARAUJO, J.; GIMÉNEZ, J. y ROSICH, N. (2006): Afectos y demostraciones geométricas en la formación inicial del docente. Enseñanza de las Ciencias, 24(3), 371-386.

BARRANTES, M. y BLANCO, L. (2006): A study of prospective primary teachers' conceptions of teaching and learning school geometry. Journal of Mathematics Teacher Education, 9 (5), 411-436. DOI: 10.1007/s10857-006-9016-6.

CALLEJA, M. F.; ORTEGA, T.; CALLEJA, I.; ÁRIAS, B. y CRESPO, M.T. (2007): Determinantes psicológicos del rendimiento académico en Matemáticas. En M. Sierra y M. F. Calleja, Estudio de evaluación de las Matemáticas en Castilla y León. Resumen de las líneas de investigación. Valladolid: Junta de Castilla y León.

CARRILLO, J.; CLIMENT, N.; CONTRERAS, L. C. y MUÑOZ-CATALÁN, C. (2007): Un modelo compartido para interpretar el desarrollo profesional de los profesores de matemáticas. Enseñanza de las Ciencias, 25(1), 33-44.

CORBALÁN, F.; GAIRÍN, J. M. y LÓPEZ, J. (1984): Las matemáticas al finalizar la E.G.B. Zaragoza: Instituto de Ciencias de la Educación - Universidad de Zaragoza.

ESTRADA, A.; BATANERO, M. C. y FORTUNY, J. M. (2005): Un estudio sobre el conocimiento de estadística elemental de profesores en formación. Educación Matemática, 16(1), 89-112.

FLORES, P. y MORENO, A. J. (2005): Familia y agentes sociales en educación matemática. Padres y Madres de Alumnos. 82, 34-38.

GAIRÍN, J. M. y MUÑOZ, J. M. (2005). El número racional en la práctica educativa: estudio de una propuesta editorial. Comunicación presentada al IX Simposio de la Sociedad Española de Investigación en Educación Matemática (SEIEM), Córdoba.

GARCÍA, M.; SÁNCHEZ, V.; ESCUDERO, I. y LLINARES, S. (2006): The dialectic relationship between research and practice in mathematics teacher education. Journal Mathematics Education, 9, 109-128.

GONZÁLEZ, M. J. y LUPIAÑEZ, J. L. (2005): ¿Qué valor social tiene el conocimiento matemático? Padres y Madres de Alumnos. 82, 29-33.

HIDALGO, S.; MAROTO, A. y PALACIOS, A. (1999): Las aptitudes básicas como elemento determinante en el rendimiento en matemáticas. Revista de Educación, 320, 271-293.

HIDALGO, S.; MAROTO, A.; ORTEGA, T. y PALACIOS, A. (2008): Estatus afectivo emocional y rendimiento escolar en matemáticas. Revista de Didáctica de las Matemáticas Uno, 1 (2), 9-28.

INCE (2000): La medida de los conocimientos y destrezas de los alumnos. Un marco para la evaluación (Proyecto internacional para la producción de indicadores de rendimiento de los alumnos. Proyecto PISA). Madrid.

INECSE (2004a): Marcos teóricos de PISA 2003. Conocimientos y destrezas en Matemáticas, Lectura, Ciencias y Solución de problemas. Madrid.

INECSE (2004b): Resumen de los primeros resultados en España. Evaluación PISA 2003. Madrid.

INECSE (2005): Pisa 2003. Pruebas de Matemáticas y solución de problemas. Madrid.

LAWSON, D. (2004): Changes in student entry competencies 1991-2001. Teaching Mathematics and Its Applications, 22(4), 171-175. 
LEÓN, M. de y RECIO, T. (2005): La presencia de las matemáticas en España. Padres y Madres de alumnos, 82, 38-41

LLINARES, S. (2008): Agendas de investigación en Educación Matemática en España. Una aproximación desde "ISI-web of knowledge" y ERIH. En Luengo, R.; Gómez, A.; Camacho, M. y Blanco, L. J. (ed.), Investigación en Educación Matemática XII. Badajoz: SEIEM y S.E.P.M. Ventura Reyes Prosper.

LLINARES, S. y VALLS, J. (2009): The building of pre-service primary teachers' knowledge of mathematics teaching: interaction and online video case studies. Instructional Science, 37(2), 247-271. (DOI 10.10007/s11251-007-9043-4).

MILGRAM, R. J. (2008): What is mathematical proficiency. En Schoenfeld, A. H. (ed.), Assesing Mathematical Proficiency.Cambridge: Cambridge University Press.

MORENO, M., MESA, G. y AZCÁRATE, C. (2007): Competencias y evaluación: desarrollo de un instrumento de análisis y caracterización de problemas matemáticos de nivel superior. Actas de Comunicaciones del XI SEIEM, La Laguna.

MULLIS, I.V.S. et al. (2002): TIMSS 2003. Marcos teóricos y especificaciones de evaluación. Madrid: Ministerio de educación cultura y deporte.

MURILLO, J. y MARCOS, G. (2009): Un modelo para potenciar y analizar las competencias geométricas y comunicativas en un entorno interactivo de aprendizaje. Enseñanza de las Ciencias, 27 (2), 241-256.

N.C.T.M. (1991): Estándares curriculares y de Evaluación para la Educación Matemática. S.A.E.M. Sevilla: Thales.

NISS, M. (2002): Mathematical competencies and the learning of mathematics: The Danish KOM project. Roskilde: Roskilde University.

OCDE (2004): The PISA 2003 Assessment Framework: Mathematics, Reading, Science and Problem Solving Knowledge and Skills. París: OCDE.

PENALVA, M. C.; ROIG, A. y RÍO, M. del (2009): Experimento de Enseñanza: tareas de aprendizaje de la geometría en la formación de maestros de Educación Infantil. En VII Jornadas de redes de Investigación en Docencia Universitaria. La calidad del proceso de enseñanza/aprendizaje universitario desde la perspectiva del cambio. Alicante: Universidad de Alicante.

PEÑAS, M. y FLORES, P. (2005): Procesos de reflexión en estudiantes para profesores de matemáticas. Enseñanza de las Ciencias, 23(1), 5-16.

PERELS, F.; GUERTLER, T. y SCHMITZ, B. (2005): Training of self-regulatory and problem-solving competence. Learning and Instruction. The Journal of the European Association for Research on Learning and Instruction (EARLI), 15(2), 123-139.

PISA (2005): Evaluación PISA 2003. Pruebas de Matemáticas y de Solución de Problemas. Madrid: MEC-INECSE.

RICO, L. (2003): Evaluación de competencias matemáticas. Proyecto PISA/OCDE 2003. En Castro, E. y Torre, E. de la (eds.), Investigación en Educación Matemática, $8^{\circ}$ Simposio de la SEIEM, Universidade da Coruña.

RICO, L. (2005a): La competencia matemática en PISA. En Fundación Santillana (Ed.), La Enseñanza de las matemáticas y el Informe PISA (pp. 21-40). Madrid: Fundación Santillana. 
RICO, L. (2005b): La alfabetización matemática y el proyecto PISA de la OCDE en España. Padres y Madres de Alumnos, 82, 7-13.

RICO, L. y LUPIÁÑEZ, J. L. (2008): Competencias matemáticas desde una perspectiva curricular. Madrid: Alianza.

SÁENZ, C. (2007): La competencia matemática (en el sentido de PISA) de los futuros maestros. Enseñanza de las Ciencias, 25(3), 355-365.

SCHOENFELD A. H. (2008): What Mathematical Proficiency. En Schoenfeld, A. H. (ed.), Assessing Mathematical Proficiency. Cambridge: Cambridge University Press.

SINGER, F. M. (2006a): A Cognitive Model for Developing a Competence-based Curriculum in Secondary Education. En Crisan, A. (ed.), Current and Future ChaIlenges in Curriculum Development: Policies, Practices and Networking for Change. Bucharest: Education 2000+ Publishers.

SINGER, F. M. (2009): Language and mathematics - distant relatives?. Disponible en: http://tsg.icme11.org/document/get/133. [Consultado: enero 2009].

SOLAR, H.; AZCÁRATE, C. y DEULOFEU, J. (2008): Una aproximación a las competencias matemáticas en interpretación de gráficas funcionales. Un estudio de caso. Comunicación presentada en el XII SEIEM, Badajoz.

SPINATH, B. y SPINATH, F. (2005): Longitudinal analysis of the link between learning motivation and competence beliefs among elementary school children. Learning and Instruction. The Journal of the European Association for Research on Learning and Instruction (EARLI), 15(2), 87-102.

WEDEGE, T. (2004): Mathematics at work. Researching adults' mathematics-containing competences. NOMAD. Nordisk Matematikkdidaktikk, 9(2), 101-122.

WINSLOW, C. (2005): Defining goals of mathematics education: the contents - competencies dialectic. Annales de Didactique et de Sciences Cognitives, 10, 131-155. 